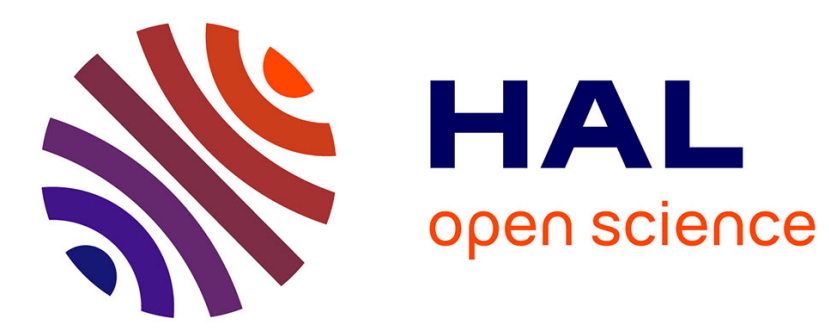

\title{
Complete game-theoretic characterization of SIS epidemics protection strategies
}

Yezekael Hayel, Stojan Trajanovski, Eitan Altman, Huijuan Wang, Piet van Mieghem

\section{- To cite this version:}

Yezekael Hayel, Stojan Trajanovski, Eitan Altman, Huijuan Wang, Piet van Mieghem. Complete game-theoretic characterization of SIS epidemics protection strategies. IEEE 53rd Annual Conference on Decision and Control (CDC 2014), Dec 2014, Los Angeles, United States. hal-01054710

\section{HAL Id: hal-01054710 https://inria.hal.science/hal-01054710}

Submitted on 8 Aug 2014

HAL is a multi-disciplinary open access archive for the deposit and dissemination of scientific research documents, whether they are published or not. The documents may come from teaching and research institutions in France or abroad, or from public or private research centers.
L'archive ouverte pluridisciplinaire HAL, est destinée au dépôt et à la diffusion de documents scientifiques de niveau recherche, publiés ou non, émanant des établissements d'enseignement et de recherche français ou étrangers, des laboratoires publics ou privés. 


\title{
Complete game-theoretic characterization of SIS epidemics protection strategies*
}

\author{
Yezekael Hayel $^{1}$, Stojan Trajanovski ${ }^{2}$, Eitan Altman ${ }^{3}$, Huijuan Wang ${ }^{2}$ and Piet Van Mieghem ${ }^{2}$
}

\begin{abstract}
Defining an optimal protection strategy against viruses, spam propagation or any other kind of contamination process is an important feature for designing new networks and architectures. In this work, we consider decentralized optimal protection strategies when a virus is propagating over a network through a Susceptible Infected Susceptible (SIS) epidemic process. We assume that each node in the network can fully protect itself from infection at a constant cost, or the node can use recovery software, once it is infected.

We model our system using a game theoretic framework. Based on this model, we find pure and mixed equilibria, and evaluate the performance of the equilibria by finding the Price of Anarchy (PoA) in several network topologies. Finally, we give numerical illustrations of our results.
\end{abstract}

\section{INTRODUCTION}

The spread of viruses in communication and computer networks or rumors in social networks can be similarly described, using epidemic models [1], [2], [3], [4]. The probability of infection over time [1], [2], [5], especially in the steady-state, and the epidemic threshold [2] in relation to the properties of the underlying network have been widely studied in the past. We consider the Susceptible Infected Susceptible (SIS) model, which is one of the mostly studied epidemic models [1], [5]. In the SIS model, at a specific time, the state of each node is either susceptible or infected. The recovery (curing) process of each infected node is an independent Poisson process with a recovery rate $\delta$. Each infected node infects each of its susceptible neighbors with a rate $\beta$, which is also an independent Poisson process. Immunization [6], [7] (via antivirus software) and quarantining [8] (via modular partitioning) prevent nodes from being infected, while additional mechanisms, like anti-spyware software or clean-up tools, could clean the virus from an infected node.

This paper considers investment games that find appropriate protection strategies against SIS virus spread. In particular, we consider a game, in which, each node (host) is a player in the game and decides individually whether or not to invest in antivirus protection. Further, if a host does not invest in antivirus protection, it remains vulnerable to the virus spread process, but can recover (e.g., by a system recovery or clean-up software). The utility or payoff of each

\footnotetext{
*The first two authors have contributed equally. This research has been supported by the EU CONGAS project (project no. 288021).

${ }^{1} \mathrm{Y}$. Hayel is with University of Avignon, Avignon, France (yezekael.hayel@univ-avignon.fr).

${ }^{2} \mathrm{~S}$. Trajanovski, H. Wang and P. Van Mieghem are with Delft University of Technology, Faculty of Electrical Engineering, Mathematics and Computer Science, P.O. Box 5031, 2600 GA Delft, The Netherlands (e-mails: \{S.Trajanovski, H.Wang, P.F.A.VanMieghem\}@tudelft.nl).

${ }^{3}$ E. Altman is with INRIA, Sophia Antipolis, France (eitan.altman@inria.fr).
}

node (player) is: (i) the investment cost, if the node decides to invest in antivirus software or else (ii) the cost of being infected, which is proportional to the infection probability in the epidemic steady-state.

Game theoretical studies for networks problems have been conducted, in routing [9], [10], network flow [11], workload on the cloud [12] or optimal network design [13], employing standard game-theoretic concepts [14], [15] such as pure Nash or mixed equilibrium. The Price of Anarchy (PoA) [16], [15] is often used as an equilibrium performance evaluation metric. While many papers have been focused on the process of virus spread and network immunizations to suppress the spread, few epidemic studies control the curing rate via game theory. Omić et al. [17] tune the strength of the nodal antivirus protection i.e. how big those (different) $\delta$ should be taken. Contrarily to [17], (i) we fix the curing and infection rates, which are not part of the game, and the decision consists of a player's choice to invest in an antivirus or not; (ii) we also consider mixed strategies Nash Equilibrium. The goal of [17] is in finding the optimal $\delta_{i}$ for each player $i$, while this paper targets the optimal decision of taking an anti-virus that fully protects the host, because today's antivirus software packages provide accurate and up to date virus protection. The related papers on security games [18], [19], [20], [21], [22] are usually applied in nonSIS environments (e.g., (i) without considering the infection state of the neighbors and (ii) without an additional mechanism for recovery), for generalized game settings [21] or by assigning nodal weights to reflect the security level [20].

Our main contributions are summarized as follows:

1) We prove that the game is a potential game by showing that it is equivalent to a congestion game. We determine a closed-form expression for the pure equilibrium for a single community/full mesh network. We also prove the existence and uniqueness of a mixed equilibrium.

2) We provide a measure of the equilibrium efficiency based on the Price of Anarchy (PoA).

3) We extend our equilibrium analysis to bipartite networks, where we show that multiple equilibria are possible. At an equilibrium, the number of nodes that invest in one partition is often close to the number of nodes that invest in the other partition.

Due to space limitations, we present the results for two, realworld motivated network topologies (single community/full mesh and bipartite network). However, results for other network topologies, convergence algorithms to the equilibria 
points and their evaluations are given in our Tech. Report [23].

The paper is organized as follows. The SIS epidemic model is introduced in Section II. Sections III and IV describe the game models in a single community (full mesh) and bipartite network, respectively and subsequently prove game theoretic results (pure, mixed equilibria and the Price of Anarchy). The conclusion is given in Section V. In order to enhance the readability, the mathematical proofs of the non-trivial prepositions and corollaries are given in the appendix.

\section{SIS EPIDEMICS ON NETWORKS}

We start with the general case, where the network $G$ is connected, undirected and unweighted with a large number $N$ of nodes. The virus behaves as an SIS epidemic, where an infected node can infect each of its direct, healthy neighbors with rate $\beta$. Each node can be cured at rate $\delta$, after which the node becomes healthy, but susceptible again to the virus. Both infection and curing process are independent Poisson processes. All nodes in the network $G$ are prone to a virus that can re-infect the nodes multiple times.

We denote the viral probability of infection for node $i$ at time $t$ by $v_{i}(N ; t)$. For each node $i$ of the graph with $N$ nodes, the SIS governing equation, under the standard $N$-Intertwined mean-field approximation (NIMFA) [24] and scaling the time as $t^{*}=\delta t$, is given by

$$
\frac{d v_{i}(N ; t)}{d t^{*}}=-v_{i}(N ; t)+\tau\left(1-v_{i}(N ; t)\right) \sum_{j=1}^{N} a_{i j} v_{j}(N ; t)
$$

where $\tau=\frac{\beta}{\delta}$ is the effective spreading rate. We further confine ourselves to the stationary regime of the SIS epidemic process, meaning $\lim _{t \rightarrow \infty} \frac{d v_{i}(N ; t)}{d t}=0$, and we denote $v_{i \infty}(N)=\lim _{t \rightarrow \infty} v_{i}(N ; t)$. The steady-state equations, valid for any graph [1], [24], are

$$
v_{i \infty}(N)=1-\frac{1}{1+\tau \sum_{j=1}^{N} a_{i j} v_{j \infty}(N)}
$$

for $i=1,2, \ldots, N$. These steady-state equations only have two possible solutions: the trivial $v_{i \infty}(N)=0$, corresponding to the exact absorbing state in SIS epidemics, and the non-trivial solution, corresponding to the metastable SIS regime.

The infection probabilities could be substantially different after some nodes decide to invest in a protection, causing those nodes not to be part of the epidemic process. Three scenarios are considered. The first one is a single community game, which could be regarded as a simple social network or a wireless and other full mesh networks (e.g., MANETs). We also study bipartite networks, often employed in the design of telecommunication networks. The main reason is that a bipartite topology provides satisfactory level of robustness after node or link failures. For instance, the topology of the Amsterdam Internet Exchange is designed as a bipartite network such that all the locations in Amsterdam are connected to two high throughput Ethernet switches. In addition, the topologies of sensor networks are also bipartite graphs.

\section{A. Single community (full mesh) network}

We first consider a single community (or full mesh) network, modeled as a complete graph $K_{N}$, where $a_{i j}=1$ for all $i$ and $j$. By symmetry, we find that $v_{i \infty}(N)=$ $v_{j \infty}(N)=v_{\infty}(N)$ for any node $i$ and $j$. From (1), we have [1], [5]

$$
v_{i \infty}(N)=\left\{\begin{array}{cl}
1-\frac{1}{\tau(N-1)}, & \text { if } \tau \geq \frac{1}{N-1}, \\
0, & \text { otherwise }
\end{array}\right.
$$

for each node $i$ in a complete graph.

\section{B. Bipartite network}

The bipartite network $K_{M, N}$ consists of two clusters with $M$ and $N$ nodes, such that one of the nodes within a cluster are connected, but all the nodes from different clusters are connected to each other Therefore, there are exactly $(M+N)$ nodes and $M N$ links in the network.

The bipartite network also possesses an interesting property, if a node is removed from the original graph from any of the two clusters, the resulting graph is again a bipartite graph either $K_{M-1, N}$ or $K_{M, N-1}$.

The governing equations (1) reduce for $K_{N, M}$ to two equations with two unknowns - the infection probabilities $v_{m \infty}$ and $v_{n \infty}$ in the two clusters in metastable state stationary regime [1], which solution is given by

$$
v_{\infty}^{m}=\frac{\tau^{2} M N-1}{\tau M(\tau N+1)} \text { and } v_{\infty}^{n}=\frac{\tau^{2} M N-1}{\tau N(\tau M+1)}
$$

\section{GAME MODEL ON A SINGLE COMMUNITY NETWORK}

In the investment game on the complete graph $K_{N}$, each node is a player and decides individually to invest in antivirus protection. The investment cost is $C$, while the infection cost is $H$. When a node invests, it is assumed to be directly immune to the virus and not part of the epidemic process anymore. Hence, this node cannot infect other nodes nor can be infected. If a node does not invest in antivirus protection, it is prone to the epidemics and might be infected by the virus (with rate $\beta$ ), but also can use additional protective mechanisms, like recovery or anti-spyware software (with rate $\delta$ ). The induced network topology, after the removal of nodes that decide to invest, determines the process of epidemic spread.

\section{A. Pure strategies}

The investment cost for any player is a constant $C$ and does not depend on the action of the other players. If a player decides not to invest, his cost is a linear function of his expected infection time $E[T]=\frac{1}{T} \int_{0}^{T} E\left[X_{i}(N ; t)\right] d t=$ $\frac{1}{T} \int_{0}^{T} \operatorname{Pr}\left[X_{i}(N ; t)=1\right] d t$, since $E\left[X_{i}\right]=\operatorname{Pr}\left[X_{i}\right]$ for Bernoulli random variables.

When the time $T$ becomes large, $E[T]$ is approximated by the infection probability $v_{i \infty}(N)$ of node $i$ in the metastable state of the SIS process. The probability $v_{i \infty}(N)$ depends explicitly on the number of nodes that decide not to invest. In other words, there is an initial contact graph $G$ in which all the nodes are connected and the decisions of all the nodes induce an overlay graph $G_{g}$ only composed of the nodes that have decided not to invest. 
1) Congestion Game: Due to players' decisions, we have a congestion game, because the utility of each player depends on the number of players that have decided not to invest. Each node has the choice between two actions: invest (further denoted by 1 ) or not (further denoted by 0 ). The payoff obtained by a player, in case he does not invest, depends on the number of players that choose the same action $(0)$ not to invest. We denote by $\sigma_{i} \in\{0,1\}$ the action of node $i$. For example, the payoff $S_{i 1}$ of a player $i \in\{1,2, \ldots, N\}$ which decides to invest is defined by: $S_{i 1}=C:=S_{1}$, while the payoff of a player $i$ which decides not to invest is: $S_{i 0}(n)=$ $H v_{i \infty}(n):=S_{0}(n)$, where $n$ is the number of players that do not invest. This game is a congestion game [25] as the payoff of a player depends on the number of players that choose his action. In the context of a congestion game, a (pure) Nash equilibrium is a vector of (pure) strategies, characterized by the number of nodes $n^{*}$ that do not invest. We remark that several Nash equilibria lead to the same $n^{*}$. We are interested in the existence and uniqueness of this value $n^{*}$.

Definition 1. At a Nash equilibrium, no node has an interest to change unilaterally his decision. The number $n^{*}$ of nodes that do not invest at a Nash equilibrium is defined for any player $i$, by: $S_{i 1} \leq S_{i 0}\left(n^{*}+1\right)$ and $S_{i 0}\left(n^{*}\right) \leq S_{i 1}$.

Our game is symmetric as all players share the same set of payoff functions. The following important property (in Preposition 1) says that our game is not only a congestion game but also a potential game, due to the potential formula in [26, Theorem 3.1].

Proposition 1. The game is potential, where $\Phi(n)=C(N-$ $n)+H \sum_{k=2}^{n} v_{\infty}(k)$ is the potential function of the game.

The existence of a potential function in a game shows the existence of pure Nash equilibrium: any minimum of the potential function $\Phi$ is a pure equilibrium. The existence also allows decentralized procedures like best response dynamics or reinforcement learning [27], [28] to converge to the pure Nash equilibrium. We can assume, for example, that an investment is valid only for a fixed amount of time and then each node pays again after expiration of his license.

Proposition 2. For the number of nodes $n^{*}$ that do not invest at equilibrium, the following inequality holds:

$$
v_{\infty}\left(n^{*}\right) \leq \frac{C}{H} \leq v_{\infty}\left(n^{*}+1\right) .
$$

Moreover, above the epidemic threshold $\left(\tau>\frac{1}{N-1}\right), n^{*}$ is uniquely defined by:

$$
n^{*}= \begin{cases}\min \left\{N,\left\lceil\frac{1}{\left(1-\frac{C}{H}\right) \tau}\right\rceil\right\}, & \text { if } C<H \\ N, & \text { otherwise }\end{cases}
$$

where $\lceil x\rceil$ is the closest integer greater or equal than $x$.

2) Performance of the equilibrium: In order to evaluate the performance of the system, considering a non-cooperative behavior of each node, we use the Price of Anarchy (PoA) metric [16]. We define the social welfare $S W(n)$ of this system, when $n$ users do not invest, as the summation of the cost for all users:

$$
S W(n)=\sum_{i=1}^{N} S_{i \sigma_{i}}(n)=C(N-n)+n H v_{\infty}(n)
$$

We define $n^{\text {opt }}$ such that: $n^{\text {opt }}=\arg \min _{n} S W(n)$, while the Price of Anarchy, considering pure strategies, is defined by:

$$
P o A_{p}=\frac{S W\left(n^{o p t}\right)}{S W\left(n^{*}\right)} \leq 1
$$

Proposition 3. The value that minimizes the social welfare is $n^{\text {opt }} \in\left\{N,\left\lceil 1+\frac{1}{\tau}\right\rceil\right\}$.

The following Corollary 1 also holds:

Corollary 1. The equilibrium value $n^{*}$ is at least as large as the optimum value $n^{\text {opt }}$, thus $n^{*} \geq n^{\text {opt }}$.

Because, we have determined $n^{*}$ and $n^{o p t}$, we can find $P o A_{p}$ in an exact, but rather complex form. We can also bound $\operatorname{PoA}_{p}$.

Corollary 2. The Price of Anarchy PoAp is bounded by:

$$
1-\left(1+\frac{1}{\tau}\right) \frac{1}{N} \leq \operatorname{Po}_{p} \leq 1 .
$$

\section{B. Symmetric mixed strategies}

We now assume that each individual decides with a probability $p$ to invest in the anti-virus protection. Moreover, the game is symmetric and then we look for a symmetric mixed Nash equilibrium. Each individual is faced with a new game, which depends on the realization of the random choice process of all the other individuals. We denote by $\bar{S}_{i}\left(\sigma_{i}, p\right)$ the expected cost of player $i$ choosing the pure strategy $\sigma_{i}$ against the probability choice $p$ of the other $N-1$ players. For any user $i$, we have $\bar{S}_{i \sigma_{i}}(p)=\sum_{n=0}^{N-1} S_{i \sigma_{i}}(n+$ 1) $\left(\begin{array}{c}N-1 \\ n\end{array}\right)(1-p)^{n} p^{N-1-n}$, where by definition $S_{i 0}(1)=0$. Hence, the total expected cost of node $i$ which invests with probability $p^{\prime}$ and when all the other nodes invest with probability $p$, is:

$$
\bar{S}_{i}\left(p^{\prime}, p\right)=p^{\prime} \bar{S}_{i 1}(p)+\left(1-p^{\prime}\right) \bar{S}_{i 0}(p) .
$$

Definition 2. At equilibrium, the indifference property $p^{*}$ is solution of $\bar{S}_{i}\left(0, p^{*}\right)=\bar{S}_{i}\left(1, p^{*}\right)$.

We now show the existence and uniqueness of a symmetric mixed equilibrium $p^{*}$.

\section{Proposition 4. A symmetric mixed equilibrium exists.}

Now, the equilibrium point $p^{*}$ could be determined from an exact, but rather complex, non-closed expression in $p$ :

$$
\begin{aligned}
\bar{S}_{i}(0, p) & =\sum_{n=0}^{N-1} S_{i 0}(n+1)\left(\begin{array}{c}
N-1 \\
n
\end{array}\right)(1-p)^{n} p^{N-1-n} \\
& =H \sum_{n=\underline{n}}^{N-1}\left(1-\frac{1}{\tau n}\right)\left(\begin{array}{c}
N-1 \\
n
\end{array}\right)(1-p)^{n} p^{N-1-n},
\end{aligned}
$$

with $\underline{n}=\left\lceil\frac{1}{\tau}\right\rceil$ because $S_{i 0}(n+1)=v_{\infty}(n+1)$ if $\tau \geq \frac{1}{n}$ and $S_{i 0}(n+1)=0$, otherwise.

Proposition 5. The symmetric mixed equilibrium is unique. 
Further, it is possible to modify (6) in a complex form involving generalized hyper-geometric function [29], which explains the difficulty of finding a closed form for $p^{*}$.

1) Approximation: In order to get a closed form expression of the symmetric mixed strategy, we consider the following approximation: instead of considering a player faced to realize a symmetric mixed strategy of the other players and optimizing his average cost, we consider that a player is part of an average game. In this case, if player $i$ chooses strategy 1 with probability $p_{i}$ we obtained the following average approximated cost: $\hat{S}_{i}\left(p_{i}, p^{\prime}\right)=p_{i} C+(1-$ $\left.p_{i}\right) H v_{i \infty}\left(\bar{n}\left(p^{\prime}\right)+1\right)$, where $\bar{n}\left(p^{\prime}\right)$ is the average number of nodes, except node $i$, that decide not to invest, i.e. $\bar{n}\left(p^{\prime}\right)=$ $\left(1-p^{\prime}\right)(N-1)$.

Proposition 6. If we approximate the number of nodes that do not invest by its average, we obtain the following symmetric mixed Nash equilibrium:

$$
\hat{p}^{*}=\left\{\begin{array}{cl}
1-\frac{H}{\tau(H-C)(N-1)}, & \text { if } C<H\left(1-\frac{1}{\tau(N-1)}\right), \\
0, & \text { otherwise. }
\end{array}\right.
$$

If the investment $\operatorname{cost} C$ is higher than the curing cost $H$, then the equilibrium is $\hat{p}^{*}=0$, because even, if a node is infected, its cost $H$ is less than the $\operatorname{cost} C$, then he would pay to be protected.

2) Performance of symmetric mixed equilibrium: The social welfare can be defined considering the mixed strategies: $S W(p)=\sum_{i=1}^{N} \bar{S}_{i}(p, p)$.

Further, we compute the optimal social welfare by using the approximated cost function $\hat{S}_{i}$ :

$$
\begin{aligned}
& \arg \min _{p} S W(p) \simeq \arg \min _{p}[p \hat{S}(1, p)+(1-p) \hat{S}(0, p)] \\
= & \arg \min _{p}\left\{\begin{array}{l}
(C-H) p+H\left(1-\frac{1}{\tau(N-1)}\right), \text { if } p \in\left[0,1-\frac{1}{\tau(N-1)}\right) \\
p C, \text { if } p \in\left[1-\frac{1}{\tau(N-1)}, 1\right] .
\end{array}\right.
\end{aligned}
$$

Proposition 7. The optimal solution of the social welfare $(S W)$ is $N \min \{C, H\}\left(1-\frac{1}{\tau(N-1)}\right)$ and it is achieved for

$$
\hat{p}^{\text {opt }}= \begin{cases}0, & \text { if } C>H \\ {\left[0,1-\frac{1}{\tau(N-1)}\right],} & \text { if } C=H \\ 1-\frac{1}{\tau(N-1)}, & \text { if } C<H\end{cases}
$$

Based on Proposition 7 and $S W\left(p^{*}\right)=N C$, we are now able to approximate $P o A_{m}$ in the case of mixed strategies:

Corollary 3. When each node uses a mixed strategy, the Price of Anarchy PoA $A_{m}$ can be approximated by:

$$
P_{o} A_{m} \approx \frac{\min \{C, H\}\left(1-\frac{1}{\tau(N-1)}\right)}{C} .
$$

\section{Comparison of strategies}

In the previous sections, we have studied two different approaches for our non-cooperative investment game: the pure and the mixed strategies. These two game variants assume significantly different decision processes for each node. First, the approximation of the expected number of nodes that do not invest at equilibrium is very close to the result obtained using the potential game approach: $\hat{n}=N\left(1-\hat{p}^{*}\right) \simeq n^{*}$. Second, we compare the social welfares obtained in each situation, and we observe that pure strategies always yields a lower social welfare compared with symmetric mixed strategies.

As stated in Corollary $3, \bar{S}_{i}\left(0, p^{*}\right)=\bar{S}_{i}\left(1, p^{*}\right)=C$, i.e. the payoff of all the players is equal, hence $S W_{m}^{*}=C N$. On the other hand, in the proof of Corollary 2, we find $S W_{p}^{*}:=$ $S W\left(n^{*}\right)>C N$. Corollary 4 immediately follows.

Corollary 4. The social welfare is smaller if all the nodes use a pure strategy $\left(S W_{p}^{*}\right)$ compared to the case in which all the nodes use a symmetric mixed strategy $\left(S W_{m}^{*}\right)$, i.e. $S W_{p}^{*}<S W_{m}^{*}$.

The bound achieved in Corollary 4 is tight, because $\left(S W\left(n^{*}\right)-C N\right)$ is small - based on Preposition 2. This is also visualized in Fig. 1a, where indirectly, by comparing the Price of Anarchy for different equilibria, we show the approximation leads to almost correct value for the real expected payoff.

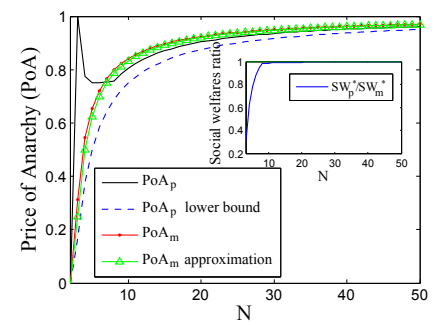

(a)

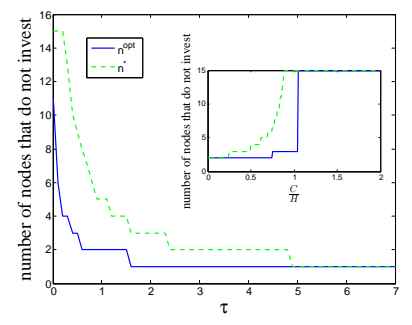

(b)
Fig. 1: (a) Price of Anarchy depending on the number of nodes $N$ (main plot). Ratio $\frac{S W_{p}^{*}}{S W_{m}^{*}}$ of the social welfares depending on the size $N$ of the network (inset); (b) Number of nodes which do not invest as a function of the effective spreading factor $\tau$ (main plot) or the ratio $\frac{H}{C}$ (inset) for $N=15$; and (c) Convergence of the RLA to the pure Nash equilibrium. Probability of 3 random nodes that invest at each iteration (main plot); number of nodes that invest after each iteration (inset).

We evaluate the performance of the decentralized system (equilibrium) compared to the centralized point of view (social optimum) via the Price of Anarchy of our system in different cases: pure and mixed strategies. We show how this metric depends on the system parameters, such as the number of nodes (decision makers), the effective epidemic spreading rate $\tau=\frac{\beta}{\delta}$ and the costs $C$ and $H$.

Fig. 1a illustrates the PoA with the following costs $C=$ $0.4, H=0.5$ and the effective spreading rate $\tau=2 / 3$. We observe that when the number of nodes is relatively small $(N<8)$, using pure strategies yields a higher PoA compared to using mixed strategies. Moreover, we find that the lower bound of the pure $P o A_{p}$ is very close to $P o A_{m}$, when $N$ becomes relatively large $(N>10)$. We also observe that the approximation of the expected payoff, which induces a closed form expression of the mixed equilibrium, is very close to the exact $\mathrm{Po}_{m}$. We show in Fig. 1a (inset) the ratio $\frac{S W_{p}^{*}}{S W_{m}^{*}}$ of the social welfares depending on the size $N$ of the 
network. Fig. 1a matches Corollary 4, i.e., the social welfare obtained using pure strategies in the game, is lower than the one obtained via symmetric mixed strategies. This difference is noticeable when the network is small but diminishes quickly (e.g., for $N=8$ the ratio is $\frac{S W_{p}^{*}}{S W_{m}^{*}}=0.9821$ ).

In Fig. 1b, we describe the number of nodes which do not invest considering the two methods: decentralized $n^{*}$ (Nash equilibrium) and the centralized case $n^{o p t}$ (social welfare), depending on the effective spreading rate $\tau$ (main plot) and ratio of the costs of not investing and investing $\frac{H}{C}$ (inset). First, we observe that our result is correct, i.e., considering a decentralized point of view, the number of nodes which invest is lower than that of the centralized point of view. This result is somewhat surprising, as in general in a decentralized system, the players are more suspicious and we would think that in our setting, more nodes would invest at equilibrium compared to the central decision. Second, those numbers are exponentially decreasing with the effective spreading rate $\tau$ : the more the infection rate $\beta$ dominates the curing rate $\delta$, more nodes decide to invest in equilibrium. On the other hand, the number of nodes increases if the relative cost of investment decreases, as expected. However, the increase is faster in a decentralized system for a fixed $\frac{H}{C}$ (Fig. 1b inset).

\section{GAME MODEL IN BIPARTITE NETWORK}

In this section, we characterize the equilibrium points, their existence and uniqueness for bipartite network $G_{M, N}$. For simplicity, we define $k=\frac{C}{H}$.

Proposition 8. The equilibrium pair $\left(n^{*}, m^{*}\right)$ exists and satisfies the following inequalities:

$$
\begin{gathered}
\forall m \in \mathcal{M}, v_{\infty}^{m^{*}}\left(n^{*}, m^{*}\right)<\frac{C}{H} \leq v_{\infty}^{m^{*}+1}\left(n^{*}, m^{*}+1\right) \text { and } \\
\forall n \in \mathcal{N}, v_{\infty}^{n^{*}}\left(n^{*}, m^{*}\right)<\frac{C}{H} \leq v_{\infty}^{n^{*}+1}\left(n^{*}+1, m^{*}\right)
\end{gathered}
$$

Moreover, above the epidemic threshold, the following hold:

1) for a given $n^{*}\left(m^{*}\right)$ there is no more than one $m^{*}$ $\left(n^{*}\right)$.

2) for any $\tau$ and $k \geq \frac{1}{2}$; or $\tau \geq \frac{(1+k)(1-2 k)}{2 k(1-k)}$ and $k<\frac{1}{2}$ : $\left|n^{*}-m^{*}\right| \leq 1$ i.e. $n^{*}$ and $m^{*}$ are either equal or consecutive integers.

$3)$ in general, it is possible to have multiple equilibria pairs such that $\left|n^{*}-m^{*}\right| \geq 2$ for some $\left(n^{*}, m^{*}\right)$.

When $n$ and $m$ players do not invest in anti-virus protection, from the first and the second partition, respectively, the social welfare is given by:

$$
\begin{aligned}
S W(n, m)= & \sum_{i=1}^{N} S_{i \sigma_{i}}(n)=C(N-n+M-m) \\
& +H\left(n v_{\infty}^{n}(n, m)+m v_{\infty}^{m}(n, m)\right) .
\end{aligned}
$$

We define the optimal pair $\left(n^{o p t}, m^{o p t}\right)=$ $\arg \min _{(n, m)} S W(n, m)$ and the Price of Anarchy in $K_{N, M}: \operatorname{Po} A:=\frac{S W\left(n^{o p t}, m^{o p t}\right)}{S W\left(n^{*}, m^{*}\right)}$.

Proposition 9. In $K_{N, M}$, the minimum (optimal) value of the social welfare is equal to $S W=\max \left\{\tau^{2} M N-1,0\right\}$. $\min \left\{\frac{C}{\tau^{2} \max \{M, N\}}, H \frac{\tau(M+N)+2}{\tau(\tau M+1)(\tau N+1)}\right\}$. In particular,
1) if $M N \leq \frac{1}{\tau^{2}}$, then $S W=0$ and $\left(n^{o p t}, m^{o p t}\right)=$ $(N, M)$.

2) if $M N>\frac{1}{\tau^{2}}, \tau \max \{M, N\} \frac{\tau(M+N)+2}{(\tau M+1)(\tau N+1)} \geq k$ then: $S W=C \frac{\tau^{2} M N-1}{\tau^{2} \max \{M, N\}}$ and $\left(n^{o p t}, m^{o p t}\right)=\left(\frac{1}{\tau^{2} M}, M\right)$ if $M>N ;\left(n^{o p t}, m^{o p t}\right)=\left(N, \frac{1}{\tau^{2} N}\right)$ if $M<N$ or both points for $M=N$.

3) if $M N>\frac{1}{\tau^{2}}, \tau \max \{M, N\} \frac{\tau(M+N)+2}{(\tau M+1)(\tau N+1)}<k$ then $S W=H \frac{\left(\tau^{2} M N-1\right)[\tau(M+N)+2]}{\tau(\tau M+1)(\tau N+1)}$ and $\left(n^{o p t}, m^{o p t}\right)=$ $(N, M)$.

Based on the results in Prepositions 8 and 9, we can now find a tight bound for the Price of Anarchy (PoA).

Corollary 5. The Price of Anarchy is bounded by:

$P O A \geq \frac{\max \left\{\tau^{2} M N-1,0\right\} \min \left\{\frac{1}{\tau \max \{M, N\}}, \frac{H(\tau(M+N)+2)}{C(\tau M+1)(\tau N+1)}\right\}}{\tau(M+N)}$.

The only used inequality in the proof of Corollary 5 is from Preposition 8, where the last well-approaches the correspondent real value. Moreover, we have the following Corollary 6.

Corollary 6. The lower bound in Corollary 5 $\frac{\max \left\{\tau^{2} M N-1,0\right\} \min \left\{\frac{1}{\tau \max \{M, N\}}, \frac{H(\tau(M+N)+2)}{C(\tau M+1)(\tau N+1)}\right\}}{\tau(M+N)}$ is smaller than $\min \left\{\frac{1}{2}, \frac{H}{C}\right\}$.

Because the bound of PoA from Preposition 5 is accurate, Corollary 6 tells us that the loss of the social welfare due to decentralized investment decision often cannot be larger than $50 \%$.

For a bipartite graph, not much could be said about the mixed equilibrium due the fact that the bipartite network is not symmetric, and players' uniform social welfare function cannot be defined.

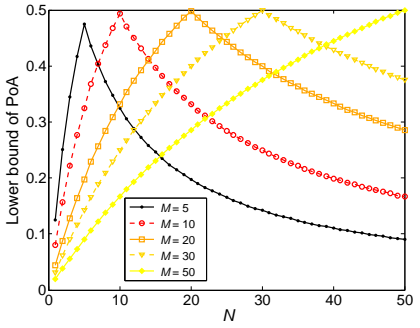

(a) $\frac{H}{C}=5$

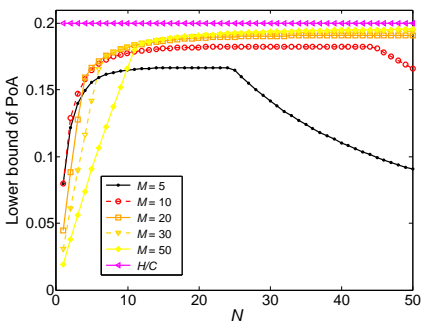

(b) $\frac{H}{C}=0.2$
Fig. 2: The lower bound of the Price of Anarchy as functions of $N$ for fixed $M$.

For the bipartite network, the lower bound of the Price of Anarchy (PoA) is illustrated in Fig. 2. In particular, Fig. 2a and $2 b$ both demonstrate the change of the lower bound of the Price of Anarchy as a function of $N$ for several fixed values of $M$. All the figures confirm Corollary 6 that the lower bound of PoA is not bigger than the smaller of $\frac{1}{2}$ or $\frac{H}{C}$. In all the cases, the closer $M$ and $N$ are to one another - the higher lower bound of PoA (maximum values in Figs. 2a and 2b). For fixed $M$ and $\frac{H}{C}>\frac{1}{2}$, the lower bound is dominated 
by $\frac{\tau^{2} M N-1}{\tau^{2}(M+N) \max \{M, N\}}$ (Corollary 5), which is a function that increases in $N$ for $N<M$, achieves its maximum (not bigger than $\frac{1}{2}$ ) and then decreases for $N>M$ (Fig. 2a). For fixed $M$ and $\frac{H}{C}<\frac{1}{2}$, the lower bound is dominated by $\frac{H}{C} \frac{\left(\tau^{2} M N-1\right)(\tau(M+N)+2)}{\tau(M+N)(\tau M+1)(\tau N+1)}$ (Corollary 5$)$, which is also a function that increases in $N$, achieves its maximum (not bigger than $\frac{H}{C}$ ) and then decreases (Fig. 2b).

\section{Conclusions}

In this paper, we explore the problem of finding optimal decentralized protection strategies in a network, where a node decides to invest in an anti-virus or to be prone to the virus SIS epidemic spread process. If a node (host) decides to invest, it cannot be infected, while if a node chooses not to invest, it can be infected by a virus and further spreads the virus inside the network. We study this problem from a game theoretic perspective. If a node decides to invest, the cost function of the node is the investment cost, otherwise the cost function is linearly proportional to the node's infection probability in the epidemic steady state.

We show the existence of a potential structure, which allows us to prove the existence and uniqueness and derive the pure and mixed equilibrium in a single-community (or mesh) network. Moreover, we find the pure equilibrium in a bipartite network. We also evaluate the performance of the equilibrium by finding the Price of Anarchy (PoA).

\section{APPENDIX}

A. Proofs of prepositions and corollaries in a single community network

Preposition 2: Let $S$ be the set of nodes that invest $C$. A node $k \notin S$ must pay an infection cost equal to $H v_{\infty}\left(n^{*}\right)$. If that node $k$ deviates ${ }^{1}$, then it will pay instead $C$. At equilibrium this user has no interest to deviate, meaning that: $C \geq H v_{\infty}\left(n^{*}\right)$.

It remains to show that nodes in $S$ do not gain by deviating. Each node in $S$ pays constant cost $C$. When deviating, a node $l$, originally in $S$, becomes connected to those not in $S$, which implies that node $l$ changes the size of the set $N \backslash S$ which becomes $n^{*}+1$. The following inequality at equilibrium also applies: $C \leq H v_{\infty}\left(n^{*}+1\right)$.

Now, we show that $n^{*}$ exists and is uniquely defined. For $C>H$, we have a trivial solution $n^{*}=N$, otherwise based on (2), we arrive at $H\left(1-\frac{1}{\left(n^{*}-1\right) \tau}\right)<C \leq H\left(1-\frac{1}{n^{*} \tau}\right)$, which gives $n^{*}=\left\lceil\frac{1}{\left(1-\frac{C}{H}\right) \tau}\right\rceil$, if $\left\lceil\frac{1}{\left(1-\frac{C}{H}\right) \tau}\right\rceil<N$, otherwise we have the trivial upper bound of $n^{*^{H}}=N$.

Preposition 3: The payoff of the nodes that do not invest is non-negative since $H \geq 0$ and $v_{\infty}(n) \geq 0$. If $n<1+\frac{1}{\tau}$, $S W(n)=C(N-n)$ which decreases in $n$. On the other hand, if $n \geq 1+\frac{1}{\tau}$, the derivative of (5) is $S W^{\prime}(n)=$ $H-C+\frac{H}{\tau(n-1)^{2}}$. Two cases can be distinguished:

1) $C<H$ : the function $S W(n)$ is strictly increasing over the interval $\left[1+\frac{1}{\tau}, N\right]$, so the minimum is achieved in $\left\lceil 1+\frac{1}{\tau}\right\rceil$.

\footnotetext{
${ }^{1}$ Deviation means that the node changes its action. If the multistrategy for the node is to invest, then "deviating" means that the node does not invest or vice versa.
}

2) $C \geq H$ : the function $S W(n)$ is increasing over the interval $\left[1+\frac{1}{\tau}, 1+\sqrt{\left.\frac{H}{\tau(C-H)}\right]}\right.$ and decreasing over $[1+$ $\left.\sqrt{\frac{H}{\tau(C-H)}}, N\right]$, so the minimum is achieved in $\left\{\left\lceil 1+\frac{1}{\tau}\right\rceil, N\right\}$ depending on the parameters of the system.

Corollary 1: If $C \geq H$, based on the proof in Proposition 2, we have $n^{*}=N$, which is clearly as large as any value of $n^{o p t} \leq N$. Otherwise $(C<H)$, based on Preposition 2: $n^{o p t}=\left\lceil 1+\frac{1}{\tau}\right\rceil$ and $S W(n)$ is increasing. Using the definition of PoA: $S W\left(n^{o p t}\right) \leq S W\left(n^{*}\right)$, which gives $n^{o p t} \leq n^{*}$.

Corollary 2: First, the denominator $S W\left(n^{*}\right)$ of $P_{o} A_{p}$ is strictly lower than $C N$. Indeed, using Preposition 2 into (5) we have: $S W\left(n^{*}\right)=C\left(N-n^{*}\right)+n^{*} H v_{\infty}\left(n^{*}\right)=C N-$ $n^{*}\left(C-H v_{\infty}\left(n^{*}\right)\right)<C N$.

In Proposition 3, we obtain $n^{o p t} \in\left\{N,\left\lceil 1+\frac{1}{\tau}\right\rceil\right\}$. If $n^{o p t}=$ $N$ then $n^{*}=N$ (Corollary 1) and $\operatorname{PoA}_{p}=1$. For the case $n^{o p t}=\left\lceil 1+\frac{1}{\tau}\right\rceil$, the following (based on Proposition 3) applies: (i) $C<H$ : the function $S W(n)$ is strictly increasing and

(ii) $C \geq H$ and $n^{o p t}=\left\lceil 1+\frac{1}{\tau}\right\rceil, S W(n)$ is also strictly increasing. Therefore, in both cases $n^{o p t}=\left\lceil 1+\frac{1}{\tau}\right\rceil \geq 1+\frac{1}{\tau}$, hence $S W\left(n^{o p t}\right) \geq S W\left(1+\frac{1}{\tau}\right)=C\left(N-\left(1+\frac{1}{\tau}\right)\right)$ i.e. $P o A_{p}=\frac{S W\left(n^{o p t}\right)}{S W\left(n^{*}\right)} \geq 1-\left(1+\frac{1}{\tau}\right) \frac{1}{N}$.

Preposition 4: For any $p \in[0,1]$ and any player $i$, we have: $\bar{S}_{i}(1, p)=C \sum_{n=0}^{N-1}\left(\begin{array}{c}N-1 \\ n\end{array}\right)(1-p)^{n} p^{N-1-n}=C$. We also have: $\bar{S}_{i}(0,0)=H\left(1-\frac{1}{(N-1) \tau}\right)>0$, and $\bar{S}_{i}(0,1)=0$.

If $C<H\left(1-\frac{1}{(N-1) \tau}\right)$ the mixed strategy $p^{*}$ exists because the function $\bar{S}_{i}(0, p)$ is continuous. Otherwise, we have for all $p \in[0,1], \bar{S}_{i}(1, p)>\bar{S}_{i}(0, p)$, meaning that the strategy 0 is dominant irrespective of the mixed strategy of the other players. In this case, the action 0 is the equilibrium.

Preposition 5: The proof relies on the monotonicity of $\bar{S}_{i}(0, p)$ and the fact that $\bar{S}_{i}(1, p)=C$ (a horizontal line), so the two curves intersect in one point. However, proving the monotonicity of $\bar{S}_{i}(0, p)$ is not trivial, because the well know function $H \sum_{n=\underline{n}}^{N-1}\left(\begin{array}{c}N-1 \\ n\end{array}\right)(1-p)^{n} p^{N-1-n}$ decreases faster than $\bar{S}_{i}(0, p)$ on some intervals of $p$, but slower on other intervals. In what follows, we prove the monotonicity of $\bar{S}_{i}(0, p)$. For simplicity, we denote $M_{n}^{N}(p)=(1-$ $\left.\frac{1}{\tau n}\right)\left(\begin{array}{c}N-1 \\ n\end{array}\right)(1-p)^{n} p^{N-1-n}$. Taking the first derivative in $p$ and using the fact that $1-\frac{1}{\tau n}<1-\frac{1}{\tau(n+1)}$, we obtain

$$
\begin{aligned}
& \frac{d\left(M_{n}^{N}(p)\right)}{d p}=(N-1)\left(1-\frac{1}{\tau n}\right)\left[C_{n}^{N-1}(p)-C_{n-1}^{N-1}(p)\right] \\
& <(N-1)\left[\left(1-\frac{1}{\tau(n+1)}\right) C_{n}^{N-1}(p)-\left(1-\frac{1}{\tau n}\right) C_{n-1}^{N-1}(p)\right]
\end{aligned}
$$

where $C_{n}^{N}(p)=\left(\begin{array}{c}N-1 \\ n\end{array}\right)(1-p)^{n} p^{N-1-n}$ (sometimes referred to as Bernstein Basis Polynomials, see e.g. [30]). Summing (8) over all $n=\bar{n}, \ldots, N-1$, multiplied by $H$ and knowing the values of the boundary term $C_{N-1}^{N-1}(p)=\left(\begin{array}{l}N-2 \\ N-1\end{array}\right)(1-p)^{N-1} p^{-1}=0$ result with $\frac{d\left(\bar{S}_{i}(0, p)\right)}{d p}=H \sum_{n=\bar{n}}^{N-1} \frac{d\left(M_{n}^{N}(p)\right)}{d p}<(N-1) H\left[\sum_{n=\bar{n}}^{N-1}(1-\right.$ $\left.\left.\frac{1}{\tau(n+1)}\right) C_{n}^{N-1}(p)-\sum_{n=\bar{n}-1}^{N-2}\left(1-\frac{1}{\tau(n+1)}\right) C_{n}^{N-1}(p)\right]=(N-$ 1) $H\left[\left(1-\frac{1}{\tau N}\right) C_{N-1}^{N-1}(p)-\left(1-\frac{1}{\tau \bar{n}}\right) C_{\bar{n}-1}^{N-1}(p)\right]=(1-$ 
$N) H\left(1-\frac{1}{\tau \bar{n}}\right) C_{\bar{n}-1}^{N-1}(p)<0$ i.e. $\bar{S}_{i}(0, p)$ is a decreasing function.

Preposition 6: Using (2), the average approximated cost is: $\hat{S}_{i}\left(p_{i}, p^{\prime}\right)=p_{i} C+\left(1-p_{i}\right) H v_{i \infty}(\bar{n}+1)=p_{i} C+$ $\left(1-p_{i}\right) H\left(1-\frac{1}{\tau\left(1-p^{\prime}\right)(N-1)}\right)$. Based on Definition 2 for the equilibrium, assuming that is achieved for $p^{\prime}=\hat{p}^{*}$, we have $\hat{S}_{i}\left(0, \hat{p}^{*}\right)=\hat{S}_{i}\left(1, \hat{p}^{*}\right)$, which gives $C=H(1-$ $\left.\frac{1}{\tau\left(1-p^{*}\right)(N-1)}\right)$. Finally, $\hat{p}^{*}=1-\frac{H}{\tau(H-C)(N-1)}$, if $C<$ $H\left(1-\frac{1}{\tau(N-1)}\right)$, otherwise $\hat{p}^{*}=0$ is an equilibrium, according to Preposition 4.

Preposition 7: First, the function is continuous in $p$, because the value is the same from the left and the right side of $1-\frac{1}{\tau(N-1)}$. If $p \in\left[1-\frac{1}{\tau(N-1)}, 1\right]$ then it is increasing for any $C$ and $H$. If $C>H$, the function is increasing on the whole interval $p \in[0,1]$, hence $\hat{p}^{\text {opt }}=0$ and the value is $H\left(1-\frac{1}{\tau(N-1)}\right)$. If $C=H$ the function is constant on $\left[0,1-\frac{1}{\tau(N-1)}\right]$, hence $\hat{p}^{\text {opt }} \in\left[0,1-\frac{1}{\tau(N-1)}\right]$ and the value is $H\left(1-\frac{1}{\tau(N-1)}\right)$. If $C<H$ the function is decreasing on $\left[0,1-\frac{1}{\tau(N-1)}\right]$, hence $\hat{p}^{o p t}=1-\frac{1}{\tau(N-1)}$ and the value is $C\left(1-\frac{1}{\tau(N-1)}\right)$.

\section{B. Proofs of prepositions and corollaries in a bipartite network}

Preposition 8: By definition, at equilibrium a user from each of the two partitions has no interest to change its pure strategy, i.e. a player that decides to invest has no interest to change its strategy. Let $S$ be the set of nodes that invest $C$. A node $k \notin S$ must pay an infection cost equal to $H v_{\infty}^{m^{*}}\left(n^{*}, m^{*}\right)$. If that node $k$ deviates, then it will pay instead $C$. At equilibrium a user from each of the partitions has no interest to deviate, meaning that: $C \geq H v_{\infty}^{m^{*}}\left(n^{*}, m^{*}\right)$ and $C \geq H v_{\infty}^{n^{*}}\left(n^{*}, m^{*}\right)$.

It remains to show that nodes in $S$ do not gain by deviating. These nodes pay $C$ each. When deviating, a node $l$ in the partition with $n^{*}$ nodes, originally in $S$ becomes connected to those not in $S$, which implies that node $l$ changes the size of $N \backslash S$ which becomes $n^{*}+1$. The following inequality at equilibrium also applies: $C \leq H v_{\infty}^{n^{*}+1}\left(n^{*}+1, m^{*}\right)$. In a similar way, considering a node in the partition with $m^{*}$ nodes, it also holds $C \leq H v_{\infty}^{m^{*}+1}\left(n^{*}, m^{*}+1\right)$.

If $k=\frac{C}{H}>1$ then for $m^{*}<M$ or $n^{*}<N$, we obtain a contradiction in the relations in the previous paragraph as $v_{\infty}^{m^{*}+1}\left(n^{*}, m^{*}+1\right)$ or $v_{\infty}^{n^{*}+1}\left(n^{*}+1, m^{*}\right)$ will be greater than 1 . Hence, the only possible value is $\left(m^{*}, n^{*}\right)=(M, N)$. Based on the discussion in the previous paragraph and the exact expression in (3) we end up with the following

$$
\begin{gathered}
m<\frac{1}{\tau(\tau n(1-k)-k)} \leq m+1 \\
n<\frac{1}{\tau(\tau m(1-k)-k)} \leq n+1
\end{gathered}
$$

Hence, we have $m=\left\lceil\frac{1}{\tau(\tau n(1-k)-k)}\right\rceil-1$ and $n=$ $\left\lceil\frac{1}{\tau(\tau m(1-k)-k)}\right\rceil-1$, from which for a given $n$, we have a unique $m$ or vice versa, which proves point 1 ) of the preposition.

Let us assume that $k \geq \frac{1}{2}$ or $\tau \geq \frac{(1+k)(1-2 k)}{2 k(1-k)}$. For simplicity in the derivations we denote $A=\tau^{2}(1-k)$ and
$B=\tau k$. From (10), we get $\frac{1}{A m-B}-1 \leq n$, hence $\frac{A}{A m-B}-$ $A-B \leq A n-B<\frac{1}{m}$. From the last inequality, we obtain $\frac{B}{A+B} \leq m(A m-B)$. Finally, $m>\frac{B+\sqrt{B^{2}+\frac{4 A B}{A+B}}}{2 A}$. Further, from (10) we obtain $A m-B<\frac{1}{n}$, hence $\frac{A\left(B+\sqrt{B^{2}+\frac{4 A B}{A+B}}\right)}{2 A}-$ $B \leq A m-B<\frac{1}{n}$ i.e. $\frac{\sqrt{B^{2}+\frac{4 A B}{A+B}}-B}{2}<\frac{1}{n} \Leftrightarrow n<$ $\frac{2}{\sqrt{B^{2}+\frac{4 A B}{A+B}}-B}=\frac{2\left(B+\sqrt{B^{2}+\frac{4 A B}{A+B}}\right)}{\frac{4 A B}{A+B}}=\frac{\left(1+\frac{A}{B}\right)\left(B+\sqrt{B^{2}+\frac{4 A B}{A+B}}\right)}{2 A}$. Hence, $n-m<\frac{A}{B} \frac{B+\sqrt{B^{2}+\frac{4 A B}{A+B}}}{2 A}=\frac{1+\sqrt{1+\frac{4 A}{B(A+B)}}}{2}=$ $\frac{1+\sqrt{1+\frac{4 \tau^{2}(1-k)}{\tau^{2} k(\tau(1-k)+k)}}}{2}=\frac{1+\sqrt{1+\frac{4(1-k)}{k(\tau(1-k)+k)}}}{2}$. The condition $k \geq \frac{1}{2}$ or $\tau \geq \frac{(1+k)(1-2 k)}{2 k(1-k)}$ is equivalent to $\frac{1-k}{k(\tau(1-k)+k)}<$ 2 , hence $n-m<\frac{1+\sqrt{1+4 \cdot 2}}{2}=2$. In the same way, $m-n<2$, hence $|n-m| \leq 1$, which completes the proof for 2). In conclusion, we find out to have limited number of possibilities to be checked $n=m ; n=m-1$ or $n=m+1$, from which the system of (9) and (10) boils down to significantly simplified one in one variable.

For 3), we give a counter example, which is not covered by the conditions in 2). Indeed, taking $\tau=\frac{1+\sqrt{40000001}}{20000} \approx$ 0.316278 and $k=\frac{\sqrt{40000001}-1}{20000} \approx 0.000316178$ give $A=$ $10^{-1}$ and $B=10^{-4}$ and the system (9) and (10) will give 6 solutions: $\left(n^{*}, m^{*}\right)=\{(1,10),(2,5),(3,3),(5,2),(10,1)\}$, where not all numbers are equal or consecutive integers.

Preposition 9: $S W(n, m)$ is a function of two variables. Bellow the epidemic threshold $\left(m n \leq \frac{1}{\tau^{2}}\right)$ and $S W(n, m)=$ $C(N+M-n-m)$. If $M N \leq \frac{1}{\tau^{2}}$, then $\left(n^{o p t}, m^{o p t}\right)=$ $(N, M)$ is the optimal pair and $S W=0$. In the remaining cases $\left(M N>\frac{1}{\tau^{2}}\right)$, because the first derivatives in both $m$ and $n$ give constant non-zero values, we look for the extremal points on the boundaries in $m, n$-plane: $m n=\frac{1}{\tau^{2}} ; m=M$ or $n=N$.

1) If $m=M$, then $n=\min \left\{N, \frac{1}{\tau^{2} M}\right\}=\frac{1}{\tau^{2} M}$ and $S W=C \frac{\tau^{2} M N-1}{\tau^{2} M}$.

2) Similarly, if $n=N$, then $m=\frac{1}{\tau^{2} N}$ and $S W=$ $C \frac{\tau^{2} M N-1}{\tau^{2} N}$. 3) If $m n=\frac{1}{\tau^{2}}$ then $\frac{1}{\tau^{2} M} \leq n \leq N$ and $S W(n)=C\left(M+N-n-\frac{1}{\tau^{2} n}\right) \cdot S W(n)$ increases to some point $\left(n=\frac{1}{\tau}\right)$ and then starts to decrease, hence the minimum is on one of the boundaries, in the same points as 1) and 2). Finally, we take the minimum of 1) and 2), which gives $S W=C \frac{\tau^{2} M N-1}{\tau^{2} \max \{M, N\}}$, achieved for $\left(n^{o p t}, m^{o p t}\right)=\left(\frac{1}{\tau^{2} M}, M\right)$ for $M>N ;\left(n^{o p t}, m^{o p t}\right)=$ $\left(N, \frac{1}{\tau^{2} N}\right)$ for $M<N$ or both points for $M=N$.

Above the epidemic threshold $\left(m n \geq \frac{1}{\tau^{2}}\right)$, we have

$$
S W(n, m)=C(N+M-n-m)+H\left(\frac{\tau^{2} m n-1}{\tau(\tau n+1)}+\frac{\tau^{2} m n-1}{\tau(\tau m+1)}\right)
$$

Taking the first derivatives and equaling them to 0 , results with

$$
\begin{aligned}
& S W_{n}^{\prime}(n, m)=-C+H\left(\frac{\tau m}{\tau m+1}+\frac{\tau m+1}{(\tau n+1)^{2}}\right)=0 \\
& S W_{m}^{\prime}(n, m)=-C+H\left(\frac{\tau n}{\tau n+1}+\frac{\tau n+1}{(\tau m+1)^{2}}\right)=0
\end{aligned}
$$


Subtracting the two equations of (11) gives $\frac{H \tau(2+(m+n) \tau)^{2}(m-n)}{(1+\tau m)^{2}(1+\tau n)^{2}}=0$. Therefore $m=n$ is the only possibility. Going back into the first equation of (11) results with $C=H$. Hence, if $C \neq H$ there is no singular point inside the region and we should again look for the extrema on the boundaries: $m n \geq \frac{1}{\tau^{2}}, n \leq N$ and $m \leq M$. 1) if $m n=\frac{1}{\tau^{2}}$, then $S W(n, m)=C(N+M-n-m)$, so we again end up with the same solution as for the case bellow the epidemic threshold, considered before.

2) if $m=M$, we have $S W(n)=C(N-$ $n)+H\left(\frac{\tau^{2} M n-1}{\tau(\tau n+1)}+\frac{\tau^{2} M n-1}{\tau(\tau M+1)}\right)$. The first derivative is $S W^{\prime}(n)=-C+H\left(\frac{\tau M}{\tau M+1}+\frac{\tau M+1}{(\tau n+1)^{2}}\right)$ and $S W^{\prime \prime}(n)=-H \frac{\tau(\tau M+1)}{(\tau n+1)^{3}}<0$. Therefore, the function could only have local maximum and we should look for the minimum on the extremal points for $\frac{1}{\tau^{2} M} \leq n \leq N$.

- $n=\frac{1}{\tau^{2} M}$ then $S W=C\left(N-\frac{1}{\tau^{2} M}\right)$ for $\left(n^{o p t}, m^{o p t}\right)=$ $\left(\frac{1}{\tau^{2} M}, M\right)$, which is again a boundary case exactly on the epidemic threshold and it was considered above.

- $n=N$ then $S W=H\left(\frac{\tau^{2} M N-1}{\tau(\tau N+1)}+\frac{\tau^{2} M N-1}{\tau(\tau M+1)}\right)=$ $H \frac{\left(\tau^{2} M N-1\right)[\tau(M+N)+2]}{\tau(\tau M+1)(\tau N+1)}$ for $\left(n^{o p t}, m^{o p t}\right)=(N, M)$.

3 ) if $n=N$, we have similar cases as in 2).

If $C=H$ (i.e. $k=1$ ) and $m=n$, then the social welfare function boils down to $S W(n)=C(N+M-2 n)+$ $2 C \frac{\tau^{2} n^{2}-1}{\tau(\tau n+1)}=C(N+M-2 n)+2 C \frac{\tau n-1}{\tau}=C\left(N+M-\frac{2}{\tau}\right)=$ const i.e. SW is constant and does not depend on $n$ or $m$. However, $C\left(N+M-\frac{2}{\tau}\right) \geq C \frac{\tau^{2} M N-1}{\tau^{2} M}$ for any $m$, because the last is equivalent to $\left(\sqrt{M}-\frac{\tau^{2} M}{\tau \sqrt{M}}\right)^{2} \geq 0$.

Corollary 5: First, the denominator of PoA: $S W\left(n^{*}, m^{*}\right)$ is strictly lower than $C(N+M)$. Indeed, using Proposition 8 and equation (7) we have: $S W\left(n^{*}, m^{*}\right)=C(N+M-$ $\left.n^{*}-m^{*}\right)+n^{*} H v_{\infty}^{n^{*}}\left(n^{*}, m^{*}\right)+m^{*} H v_{\infty}^{m^{*}}\left(n^{*}, m^{*}\right)=C(N+$ $M)-n^{*}\left(C-H v_{\infty}^{n^{*}}\left(n^{*}, m^{*}\right)\right)-m^{*}\left(C-H v_{\infty}^{m^{*}}\left(n^{*}, m^{*}\right)\right)<$ $C(N+M)$, which is an upper bound for $S W\left(n^{*}, m^{*}\right)$ and $S W\left(n^{o p t}, m^{o p t}\right)$ could be determined exactly based on Preposition 9, which completes the proof.

Corollary 6: For simplicity, we denote $h(M, N, \tau)=$ $\frac{\max \left\{\tau^{2} M N-1,0\right\} \min \left\{\frac{1}{\tau \max \{M, N\}}, \frac{H(\tau(M+N)+2)}{C(\tau M+1)(\tau N+1)}\right\}}{\tau(M+N)}$.

$\tau^{2} M N \leq 1$, then $h(M, N, \tau)=0 \leq \min \left\{\frac{1}{2}, \frac{H}{C}\right\}$. If $\tau^{2} M N>1$, then $h(M, N, \tau) \leq \frac{\tau^{2} M N-1}{\tau^{2} \max \{M, N\}(M+N)}=$ $\frac{1-\frac{1}{\tau^{2} M N}}{\max \{M, N\}\left(\frac{1}{M}+\frac{1}{N}\right)}=\frac{1-\frac{1}{\tau^{2} M N}}{1+\frac{\max \{M, N\}}{\min \{M, N\}}}<\frac{1-0}{1+1}=\frac{1}{2}$ and

$h(M, N, \tau) \leq \quad \frac{H}{C} \frac{\left(\tau^{2} M N-1\right)(\tau(M+N)+2)}{\tau^{2} M N(\tau M+1)(\tau N+1)}$
$\frac{H}{C} \frac{\tau^{2} M N(M+N)-\tau M-\tau N-2}{\tau^{2} M N(M+N)+\tau M(M+1)+\tau N(N+1)}<\frac{H}{C}$.

\section{REFERENCES}

[1] P. Van Mieghem, J. Omić, and R. Kooij, "Virus spread in networks," IEEE/ACM Trans. on Netw., vol. 17, no. 1, pp. 1 -14, feb. 2009.

[2] D. Chakrabarti, Y. Wang, C. Wang, J. Leskovec, and C. Faloutsos, "Epidemic thresholds in real networks," ACM Trans. Inf. Syst. Secur, vol. 10 , no. 4, pp. 1:1-1:26, Jan. 2008 .

[3] A. Ganesh, L. Massoulie, and D. Towsley, "The effect of network topology on the spread of epidemics," in IEEE INFOCOM, vol. 2, 2005, pp. 1455-1466 vol. 2.

[4] J. Kephart and S. White, "Directed-graph epidemiological models of computer viruses," in IEEE Symposium on Research in Security and Privacy, 1991, pp. 343-359.
[5] P. Van Mieghem, "The viral conductance of a network," Computer Communication, vol. 35, no. 12, pp. 1494-1506, Jul. 2012.

[6] R. Cohen, S. Havlin, and D. ben Avraham, "Efficient immunization strategies for computer networks and populations," Phys. Rev. Lett., vol. 91, p. 247901, Dec 2003.

[7] C. M. Schneider, T. Mihaljev, S. Havlin, and H. J. Herrmann, "Suppressing epidemics with a limited amount of immunization units," Phys. Rev. E, vol. 84, p. 061911, Dec 2011.

[8] J. Omić, J. Martín-Hernández, and P. Van Mieghem, "Network protection against worms and cascading failures using modularity partitioning," in International Teletraffic Congress (ITC), Sept 2010, pp. $1-8$.

[9] A. Orda, R. Rom, and N. Shimkin, "Competitive routing in multiuser communication networks," IEEE/ACM Trans. Netw., vol. 1, no. 5, pp. 510-521, Oct. 1993.

[10] E. Altman, T. Jimenez, T. Basar, and N. Shimkin, "Competitive routing in networks with polynomial cost," in IEEE INFOCOM, vol. 3, 2000, pp. $1586-1593$ vol.3.

[11] Y. A. Korilis and A. A. Lazar, "On the existence of equilibria in noncooperative optimal flow control," JACM, vol. 42, no. 3, pp. 584613, May 1995.

[12] A. Nahir, A. Orda, and D. Raz, "Workload factoring with the cloud: A game-theoretic perspective," in IEEE INFOCOM, 2012, pp. 25662570.

[13] A. Nahir, A. Orda, and A. Freund, "Topology design of communication networks: A game-theoretic perspective," IEEE/ACM Trans. on Netw. (to appear)

[14] E. Koutsoupias and C. Papadimitriou, "Worst-case equilibria," Computer Science Review, vol. 3, no. 2, pp. 65 - 69, 2009.

[15] T. Roughgarden and E. Tardos, "How bad is selfish routing?" JACM, vol. 49, no. 2, pp. 236-259, Mar. 2002.

[16] N. Nisan, T. Roughgarden, E. Tardos, and V. V. Vazirani, Algorithmic Game Theory. Cambridge University Press, 2007.

[17] J. Omić, A. Orda, and P. Van Mieghem, "Protecting against network infections: A game theoretic perspective," in IEEE INFOCOM, 2009, pp. 1485-1493.

[18] J. Aspnes, K. Chang, and A. Yampolskiy, "Inoculation strategies for victims of viruses and the sum-of-squares partition problem," Journal of Computer and System Sciences, vol. 72, no. 6, pp. 1077 - 1093, 2006.

[19] D. Acemoglu, A. Malekian, and A. Ozdaglar, "Network security and contagion," MIT/LIDS intern report, Tech. Rep., 2013. [Online]. Available: http://web.mit.edu/asuman/www/ documents/networksecurity-submit.pdf

[20] L. Jiang, V. Anantharam, and J. Walrand, "Efficiency of selfish investments in network security," ser. NetEcon. ACM, 2008, pp. $31-36$.

[21] M. Lelarge and J. Bolot, "Network externalities and the deployment of security features and protocols in the internet," in SIGMETRICS. ACM, 2008, pp. 37-48.

[22] M. Kearns and L. E. Ortiz, "Algorithms for interdependent security games," in In Advances in Neural Information Processing Systems. MIT Press, 2004.

[23] S. Trajanovski, Y. Hayel, E. Altman, H. Wang, and P. Van Mieghem, "Decentralized Protection Strategies against SIS Epidemics in Networks," Delft University of Technology, Tech. Rep. 20140320, March 2014. [Online]. Available: http://www.nas.ewi.tudelft.nl/people/ Stojan/TRsisprotection.pdf

[24] P. Van Mieghem, "The N-intertwined SIS epidemic network model," Computing, vol. 93, no. 2-4, pp. 147-169, 2011.

[25] R. Rosenthal, "A class of games possessing pure-strategy nash equilibria," Intl. Journal of Game Theory, vol. 2, no. 1, pp. 65-67, 1973.

[26] D. Monderer and L. S. Shapley, "Potential games," Games and Economic Behavior, vol. 14, no. 1, pp. 124 - 143, 1996.

[27] Y. Chen and R. Gazzale, "When does learning in games generate convergence to nash equilibria? the role of supermodularity in an experimental setting," American Economic Review, vol. 94, no. 5, pp. 1505-1535, September 2004

[28] R. Cominetti, E. Melo, and S. Sorin, "A payoff-based learning procedure and its application to traffic games," Games and Economic Behavior, vol. 70, no. 1, pp. 71-83, September 2010.

[29] G. Gasper and M. Rahman, Basic Hypergeometric Series, ser. Encyclopedia of Mathematics and its Applications. Cambridge University Press, 2004.

[30] G. Lorentz, Bernstein polynomials. University of Toronto Press, 1953. 